\title{
Control-Based Continuation of Nonlinear Structures Using Adaptive Filtering
}

\author{
Gaëtan Abeloos $^{1}$, Ludovic Renson ${ }^{2}$, Christophe Collette ${ }^{1,3}$, and Gaëtan Kerschen ${ }^{1}$ \\ ${ }^{1}$ Department of Aerospace \& Mechanical Engineering \\ University of Liège, Place du 20-Août 7, B-4000 Liège, Belgium \\ ${ }^{2}$ Department of Mechanical Engineering \\ Imperial College London, South Kensington Campus, London SW7 2AZ, UK \\ ${ }^{3}$ Department of Bio, Electro and Mechanical Systems \\ Université libre de Bruxelles, Avenue Franklin Roosevelt 50, 1050 Brussels, Belgium
}

\begin{abstract}
Control-Based Continuation uses feedback control to follow stable and unstable branches of periodic orbits of a nonlinear system without the need for advanced post-processing of experimental data. CBC relies on an iterative scheme to modify the harmonic content of the control reference and obtain a non-invasive control signal. This scheme currently requires to wait for the experiment to settle down to steady-state and hence runs offline (i.e. at a much lower frequency than the feedback controller). This paper proposes to replace this conventional iterative scheme by adaptive filters. Adaptive filters can directly synthesize either the excitation or the control reference adequately and can operate online (i.e. at the same frequency as the feedback controller). This novel approach is found to significantly accelerate convergence to non-invasive steady-state responses to the extend that the structure response can be characterized in a nearly-continuous amplitude sweep. Furthermore, the stability of the controller does not appear to be affected.
\end{abstract}

Keywords: Control-Based Continuation, Active Filtering, Non-Invasive Control, Nonlinear Vibrations, Experimental Characterization

\section{State of the art of Control-Based Continuation}

The characterization of a nonlinear structure consists in determining its behavior under harmonic excitation. Nonlinear structures can reach different periodic orbits under identical excitation, each one with its own response amplitude and stability. Some of the responses are unstable and cannot be observed experimentally without diverging towards another periodic orbit. Control-Based Continuation (CBC) is an experimental method that stabilizes the structure using feedback control to reach these unstable response branches [1][2]. Characterizing the unstable branches is useful to uncover potential hidden branches that, even though stable, cannot be reached by performing uncontrolled frequency sweeps [3].

Nonlinear structures respond to monoharmonic excitation with multiharmonic responses. These multiple harmonics are fed back through the controller into the excitation signal, making it multiharmonic. As such, the response of the structure is different than under monoharmonic excitation and the control is considered invasive [4]. An important part of experimental continuation procedures is to ensure that the controller is non-invasive and that the total excitation signal (control action included) is monoharmonic, i.e. that its non-fundamental harmonics are canceled. 


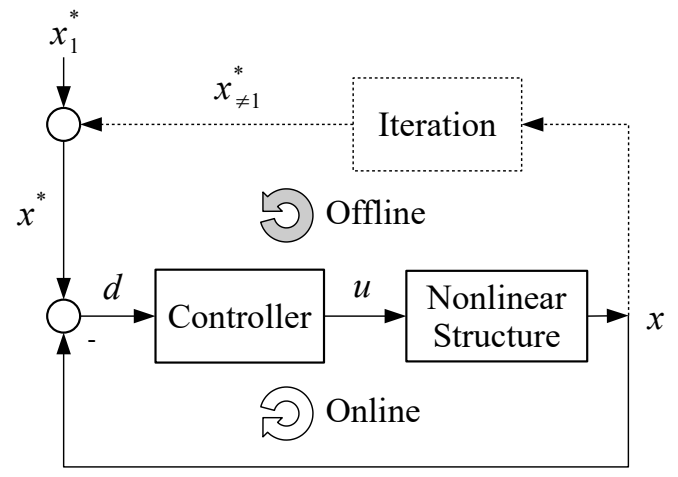

Fig. 1 Implementation of Control-Based Continuation with the online feedback loop and the offline iteration loop: the excitation $u$ is the output of the controller being fed with the difference $d$ between the target response $x^{*}$ and the measured response $x$; the target comprises a monoharmonic part $x_{1}^{*}$ determined by the user and non-fundamental harmonics $x_{\neq 1}^{*}$ resulting from Picard iterations on $x$

One way to render the controller non-invasive is to ensure that the non-fundamental harmonics of the target $x^{*}$ cancel the ones of $x$. In this way, the difference $d=x^{*}-x$ fed into the controller is monoharmonic and so is the controller output: the excitation is monoharmonic and the controller is non-invasive. Fig. 1 shows the current implementation of CBC [4][5][6]. The method consists in two loops. One online feedback loop with a controller generating the excitation signal $u$ from the difference $d$. The other loop adds to the target's fundamental harmonic $x_{1}^{*}$ the non-fundamental harmonics $x_{\neq 1}^{*}$. These are currently defined offline, for instance with Picard iterations. This is repeated until the non-fundamental harmonics of $x^{*}$ cancel the ones of $x$, making $d$ and $u$ monoharmonic. The continuation parameter is the amplitude of $x_{1}^{*}$.

\section{Control-Based Continuation with adaptive filtering}

Adaptive filters have been used for online harmonic elimination in the literature [7]. The contrary is done in this work: they are used for harmonic selection in order to replace the offline loop shown in Fig. 1 and have a fully online

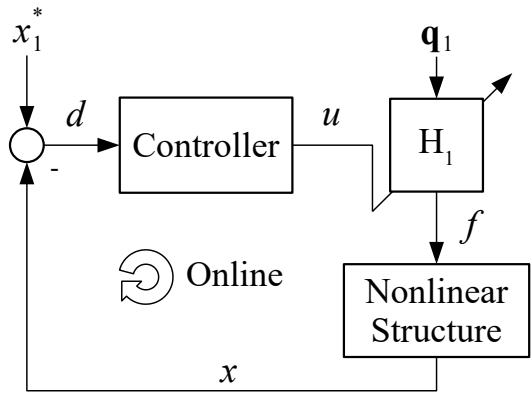

(a) The adaptive filter $\mathrm{H}_{1}$ synthesizes a monoharmonic excitation signal $f$ using the multiharmonic controller output $u$ as a reference; the input $\mathbf{q}_{1}$ contains the fundamental harmonic

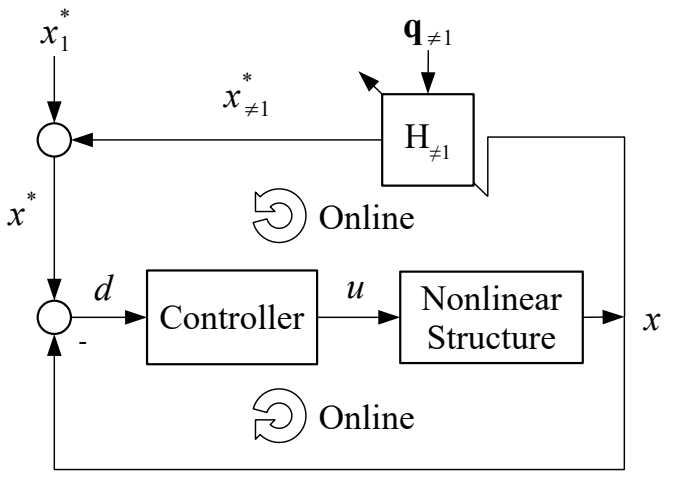

(b) The adaptive filter $\mathrm{H}_{\neq 1}$ synthesizes the nonfundamental harmonics $x_{\neq 1}^{*}$ of $x^{*}$ using $x$ as a reference; the input $\mathbf{q} \neq 1_{1}$ contains every harmonic except the fundamental

Fig. 2 Two different architectures using adaptive filters to remove the offline iteration loop, giving fully online systems; the diagonal arrows behind the filters $\mathrm{H}_{1}$ and $\mathrm{H}_{\neq 1}$ mark the reference signals 
system. The filter uses input signals q, comprising orthogonal sine waves of specific harmonics' frequencies that are combined to synthesize an output signal. The RLS algorithm minimizes the squared error between this output signal and a reference signal. This algorithm combines good performance with the ability to function with transient signals.

Fig. 2a shows a system where an adaptive filter is used to synthesize a monoharmonic excitation signal $f$ using the multiharmonic controller output $u$ as reference. In this way, $d$ and $u$ can be multiharmonic while the system is excited monoharmonically. The response target $x_{1}^{*}$ does not have to cancel the non-fundamental harmonics of $x$ and thus can be monoharmonic. Fig. 2b shows a fully online system using the same strategy as the one shown in Fig. 1. The adaptive filter is used to synthesize the non-fundamental harmonics $x_{\neq 1}^{*}$ of the target using $x$ as a reference. Consequently, $x_{\neq 1}^{*}$ cancels the non-fundamental harmonics of $x$.

With these methods, no offline iteration is needed on the control target to ensure controller non-invasiveness, avoiding waiting for steady-state between each iteration. Traditional continuation by increasing the amplitude of $x_{1}^{*}$ with finite steps is still possible but not necessary anymore. The amplitude of $x_{1}^{*}$ can be swept continuously and all responses of the structure to constant frequency harmonic excitation will be explored in full transient, removing the need to wait for steady-state altogether.

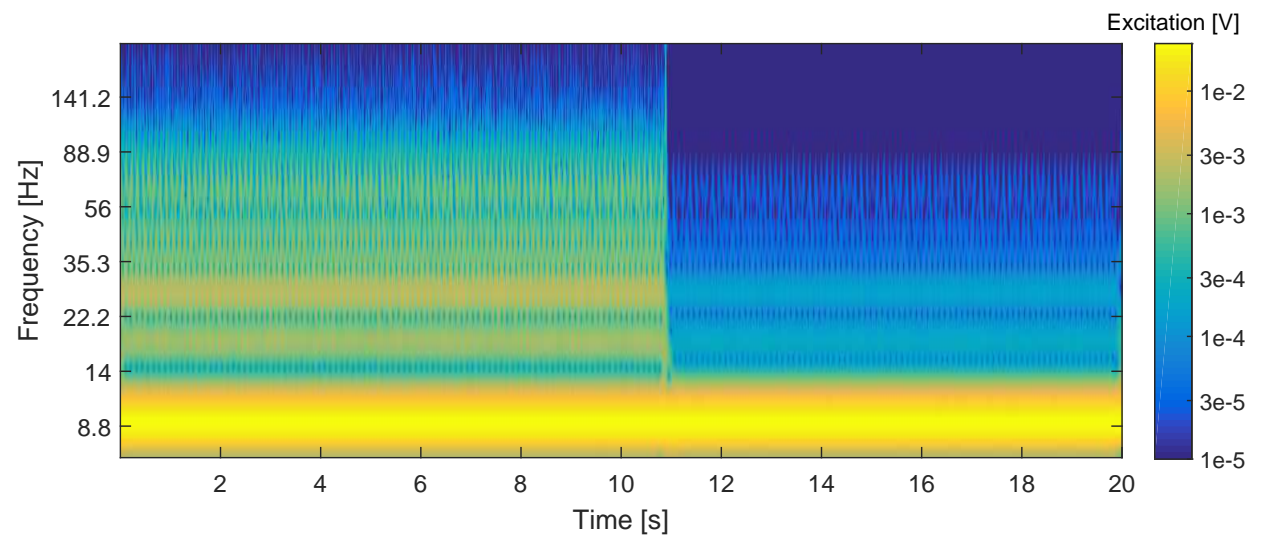

(a) The non-fundamental harmonics of the excitation signal are reduced by an order of magnitude after activation of the adaptive filter at $11 \mathrm{~s}$

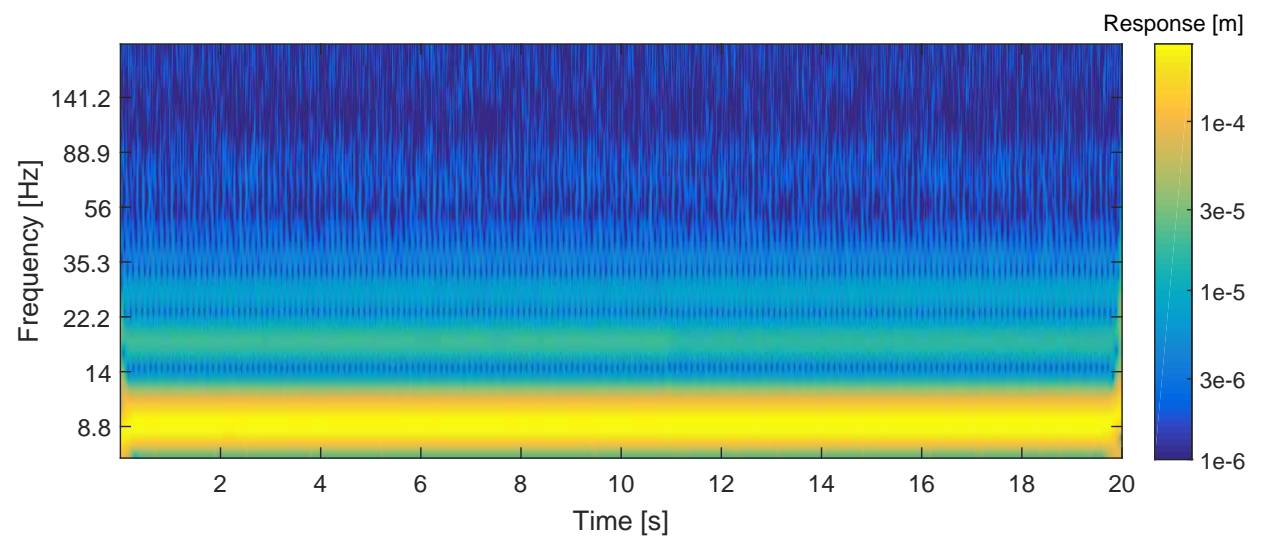

(b) The response signal is unchanged after activation of the adaptive filter at $11 \mathrm{~s}$

Fig. 3 A softening-hardening structure is stabilized at an unstable periodic orbit with CBC; before activation of the filter at $11 \mathrm{~s}$, the controller is invasive and the excitation is multiharmonic; after filter activation, the controller becomes non-invasive without destabilizing the structure from its periodic orbit 


\section{Experimental results}

The system shown in Fig. 2a was tested on a softening-hardening single degree-of-freedom energy harvester [8]. Control-based continuation was used to stabilize the system at an unstable periodic orbit to show that the filter has not significatly affected controller stabilization. Fig. 3a shows rich harmonic content in the excitation signal $f$ fed into the shaker before the filter is activated. After $11 \mathrm{~s}$, the filter is activated and the excitation becomes monoharmonic. The structure stays at the same unstable periodic orbit but the controller becomes non-invasive, as shown in Fig. 3b. With the functionality of the filtering being shown, the full response manifold of the structure can be drawn by successive full-transient sweeps of response amplitude at constant frequency.

\section{Conclusion}

A method for online canceling of the feedback controller invasiveness has been introduced. Adaptive filters are used to either synthesize a monoharmonic excitation signal, or to synthesize a control target canceling the non-fundamental harmonics harmonics fed into the controller. This method removes the need to make offline iterations on the control target while performing control-based continuation. It opens the possibility of exploring the structural responses with full-transient sweeps of the control target amplitude rather than finite continuations steps, accelerating the structural characterization procedure.

\section{Acknowledgements}

G.A. is funded by the FRIA grant of the Fonds National de la Recherche Scientifique (FNRS), L.R. is funded by a Research Fellowship from the Royal Academy of Engineering (RF1516/15/11). They gratefully acknowledge the financial support of the Royal Academy of Engineering and the FNRS.

\section{References}

[1] Sieber, J. and Krauskopf, B., Control based bifurcation analysis for experiments, Nonlinear Dynamics, Vol. 51, pp. 356-377, 2008.

[2] Sieber, J., Gonzalez-Buelga, A., Neild, S. A., Wagg, D. J. and Krauskopf, B., Experimental continuation of periodic orbits through a fold, Physical Review Letters, Vol. 100, pp. 244101, 2008.

[3] Renson, L., Shaw, A. D., Barton, D. A. W. and Neild, S. A., Application of control-based continuation to a nonlinear structure with harmonically coupled modes, Mechanical SYstems and Signal Processing, Vol. 120, pp. 449-464, 2019.

[4] Barton, D. A. W. and Sieber, J., Systematic experimental exploration of bifurcations with noninvasive control, Physical Review E, Vol. 87, pp. 052916, 2013.

[5] Renson, L., Gonzalez-Buelga, A., Barton, D. A. W. and Neild, S., Robust identification of backbone curves using control-based continuation, Journal of Sound and Vibration, Vol. 367, pp. 145-158, 2016.

[6] Renson, L., Barton, D. A. W. and Neild, S., Experimental tracking of limit-point bifurcations and backbone curves using control-based continuation, International Journal of Bifurcation and Chaos, Vol. 27, pp. 1730002, 2017.

[7] Blasko, V., A novel method for selective harmonic elimination in power electronic equipment, IEEE Transactions on Power Electronics, Vol. 22, pp. 223-228, 2007.

[8] Barton, D. A. W. and Burrow, S. G., Numerical Continuation in a Physical Experiment: Investigation of a Nonlinear Energy Harvester, Journal of Computational and Nonlinear Dynamics, Vol. 6, pp. $011010,2001$. 